Tohoku J. exp. Med., 1988, 156, 1-6

\title{
A Case of Giant Mediastinal Leiomyoma with Long-Term Survival
}

\author{
Akira Uno, Masanori Sakurai, Kikuo Onuma, Yoshio \\ Yamane, Kenkichi Kurita, Izumi Hayashi, Michiaki \\ Ikeda, Noboru Hagiwara, Kunihiko Tominaga* and \\ HaNdOU HaKOZAKI* \\ Department of Respiratory Disease and *Pathology \\ Division, Iwaki Kyoritsu General Hospital, Iwaki 973
}

Uno, A., Sakurai, M., Onuma, K., Yamane, Y., Kurita, K., Hayashi, I., Ineda, M., Hagiwara, N., Tominaga, K. and Hakozaki, H. A Case of Giant Mediastinal Leiomyoma with Long-Term Survival. Tohoku J. exp. Med., 1988, 156 (1), 1-6 — A 67-year-old female was emergently admitted to our hospital because of severe dyspnea with cyanosis. One month before admission, she consulted a doctor and chest $\mathrm{x}$-ray disclosed a huge tumor shadow occupying most of the left hemithorax. At that time, she did not complain of any particular symptoms except slight cough. Twelve days after admission, the patient lost consciousness and an emergency operation was performed. The tumor showed severe adhesion to the aorta and the lower lobe of the left lung, but not to the esophagus. After the operation, mechanical ventilation was necessary for 13 days to recover pulmonary function. Histological diagnosis was made as leiomyoma, which is rarely seen in the mediastinal region. At present, the patient survived 10 years without any symptom of recurrence. In this paper, the authors report a case of mediastinal leiomyoma which received an emergency operation and attained long term survival. Twelve similar cases reported in the past were also reviewed. -mediastinal leiomyoma; emergency operation; long-term survival

Mediastinal masses have interesting diagnostic and therapeutic problems for the clinician. Recent advances in radiographic, surgical and anesthesia techniques have improved clinical understanding and therapeutic results for mediastinal tumors. However, there are still considerable cases that definite diagnosis is difficult before surgery. Leiomyoma of mediastinum origin is quite rare in incidence (Morrison 1958; Petrikova 1982). Clinical features, including initial symptoms, radiographic appearance, and therapeutic results are still unclear. In this paper, the authors report a case with giant mediastinal leiomyoma which received an emergency operation and attained long-term disease-free survival.

There were 12 cases of mediastinal leiomyoma reported in the literature, 7 from other countries and 5 from Japan (Nishimoto et al. 1957; Rasaretnam and Panabokke 1975; Suzuki et al. 1982). A review of these cases, with special 
attention to Japanese cases, has been performed.

\section{A Case Report}

A 67-year-old female was emergently admitted to our hospital because of severe dyspnea with cyanosis. A few months before admission, she began to feel loss of appetite, following prominent weight loss. One month before admission, she consulted a doctor and a huge mass lesion in she left lung was discovered by chest x-ray (Fig. 1). She did not complain of any obvious symptoms due to the enormous shadow other than slight cough.

On admission, the patient showed clear consciousness, complaining severe dyspnea. Respiratory sound was hardly audible in the left lung. Systolic murmur of Levine 3 was audible at the trunk of the pulmonary artery. Marked tracheal deviation to the right was demonstrated by endoscopical examination. The liver, spleen and kidney were not palpable.

Blood gas analysis on admission showed $45.8 \mathrm{mmHg}$ in $\mathrm{Pa}_{\mathrm{O}_{2}}$ and $57.8 \mathrm{mmHg}$ in $\mathrm{Pa}_{\mathrm{CO}_{2}}$ in room air. Vital capacity was $450 \mathrm{ml}$. There was no abnormality in hematological examination, including electrolytes balance and liver function test. However, as the days passed, the patient was getting drowsy. Twelve days after admission, an emergent operation had to be performed because of complete loss of consciousness.

At first, the thorax was opened by left posterolateral thoracotomy at the left fifth rib. However, it was necessary to remove the ribs from fifth to eighth for complete extraction of the large tumor. The tumor originated from the anterior mediastinum and strong adhesion to both the ascending aorta and the lower lobe of the left lung was observed. The operation was finished safely with the total amount of hemorrhage of $1,774 \mathrm{ml}$. The tumor weight was $1880 \mathrm{~g}$.

It was necessary to use mechanical ventilation for 13 days after the operation to recover

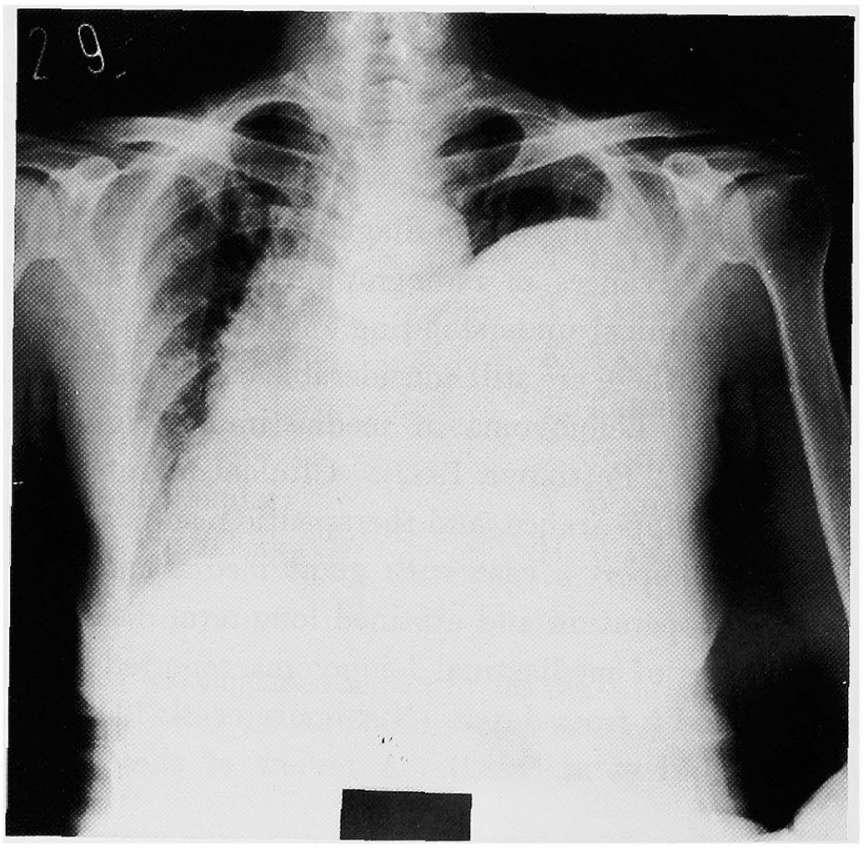

Fig. 1. Chest x-ray on admission. It revealed a huge mass lesion occupying most of the left hemithorax. 


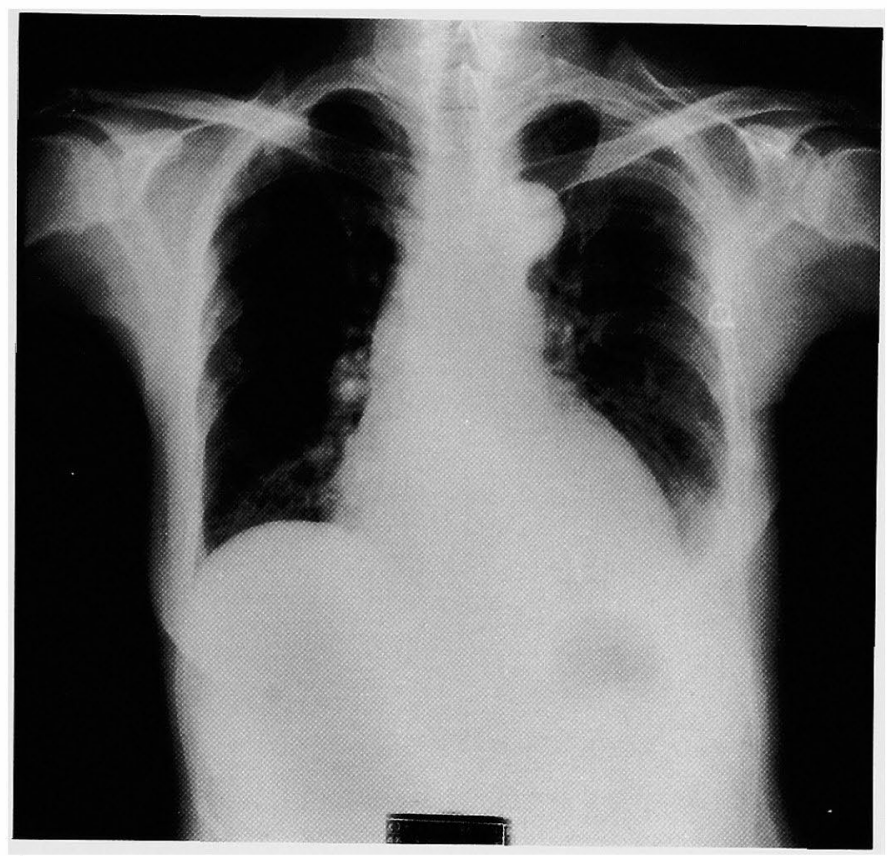

Fig. 2. Chest $\mathrm{x}$-ray just before discharge. Seventy days after operation, the patient was discharged without any operative complication.

pulmonary function. However, 70 days after operation, she was discharged without any operative complication (Fig. 2). The blood gas analysis just before discharge showed 69.2 in $\mathrm{Pa}_{\mathrm{O}_{2}}$ and 47.2 in $\mathrm{Pa}_{\mathrm{CO}_{2}}$ in room air.

\section{Pathological Findings}

The resected mass, greish-white, is $25.0 \times 21.0 \times 39.0 \mathrm{~cm}^{3}$ in size and $1880 \mathrm{~g}$ in weight covered with thin fibrous capsule (Fig. 3). The tumor has a characteristic whorl-like trabeculated appearance on cut surface. Microscopically, as shown in Fig. 4, the tumor is composed of interlacing bundles of fusiform cells, with long processes and nuclei with blunted ends, bearing a very striking resemblance to uterine myoma. Prominent cellular atypism or mitotic figures are absent. Masson's trichrom stain of the tumor tissue demonstrated myofibrils or nerve fibers but neither nuclear palisading arrangement nor Verocay bodies are observed. The tumor is rich in reticulin fibrils running parallel to the tumor cells, and in certain areas, individual tumor cells are surrounded by reticulin fibrils. The tumor tissue was revealed to be vimentin-positive and S-100 protein-negative by immunohistochemical stain.

\section{Discussion}

In this paper, a case of giant mediastinal leiomyoma was described. There have been a few reports of mediastinal tumors of smooth muscle origin. The 


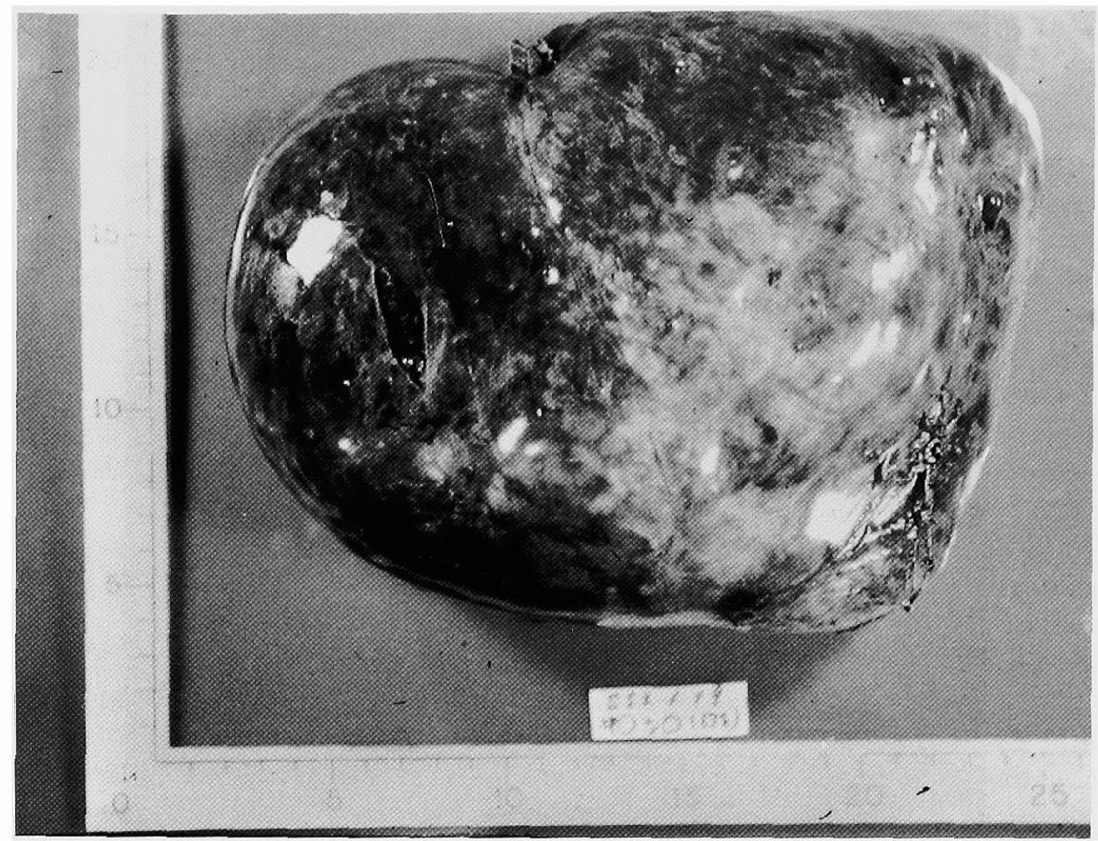

Fig. 3. Macroscopic appearance of the tumor.

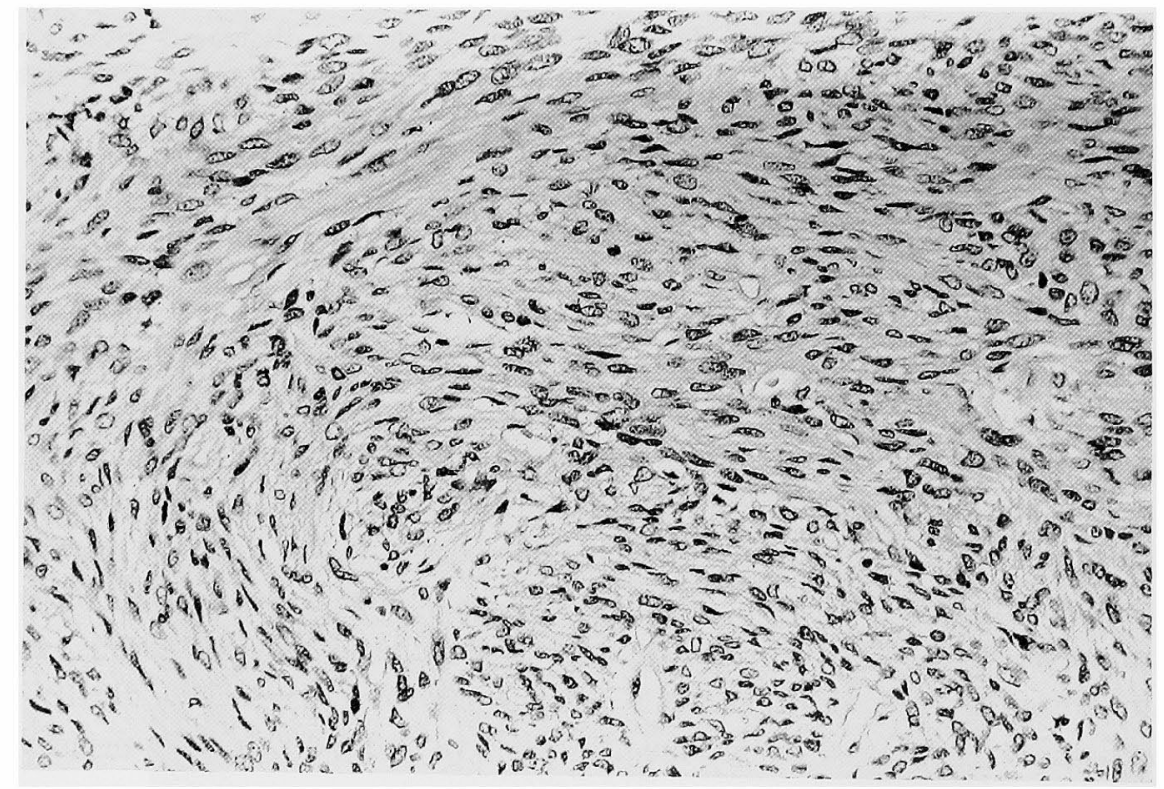

Fig. 4. Microscopic appearance of the tumor. 
A Case of Mediastinal Leiomyoma with Long-Term Survival

TABLE 1. Clinical features in 6 cases of leiomyoma of the mediastinum

\begin{tabular}{lcclrl} 
& Sex & $\begin{array}{c}\text { Age } \\
\text { (years) }\end{array}$ & \multicolumn{1}{c}{ Symptom } & $\begin{array}{c}\text { Tumor } \\
\text { weight }(\mathrm{g})\end{array}$ & \multicolumn{1}{c}{ Site } \\
\hline 1 & $\mathrm{~F}$ & 22 & Difficulty in swallowing & 34 & Posterior \\
2 & M & 47 & Feeling of weakness & - & Middle-posterior \\
3 & F & 50 & Chest pain & 200 & Posterior \\
4 & F & 42 & Dyspnea & 1600 & Posterior \\
5 & F & 46 & Chest pain & 520 & Posterior \\
$6^{*}$ & F & 67 & Dyspnea & 1880 & Anterior \\
\hline
\end{tabular}

*Present case.

M, male ; F, female.

majority of cases reported in the past usually have arisen from esophageal muscle. Rasaretnam and Panabokke reported a case of leiomyosarcoma of mediastinum with a review of 9 cases of true mediastinal tumors of smooth muscle origin, 7 of these being benign and 2 malignant (Rasaretnam and Panabokke 1975). In Japan, 5 cases of mediastinal leiomyoma have been reported (Nishimoto et al. 1957; Suzuki et al. 1982).

Regarding the clinical features of 13 benign cases ( 7 foreign, 6 Japan) including the present case, there are no characteristic symptoms attributable to this tumor, chest pain and dyspnea being common. In the 6 cases reported in Japan (Table 1), 3 cases remained free from any symptom until tumor weight reached more than $500 \mathrm{~g}$, suggesting the nature of latent progression. Age ranges from 22 to 67 , showing the similarity to those of the cases reported by Rasaretnam and Panabokke (1975). However, there is a tendency of female predominance in incidence (male : female $=1: 5$ ). As to the original site, ten of 13 cases arose from the posterior mediastinum and 3 cases from the middle mediastinum. In this point, the present case is quite rare in arising from the anterior mediastinum.

Clinical course of smooth muscle tumors of the mediastinum after resection is unclear. In the paper by Rasaretnam and Panabokke (1975) all of 3 cases of leiomyosarcoma showed local and distal recurrence within 2 years. However, there is no precise description of the clinical course of leiomyoma. Our case would be the first that has been followed-up for 10 years after resection, and at present, the patient is tumor-free, suggesting favorable prognosis, even if the tumor size is very large at operation.

Recently, Davis et al. (1987) reported an analysis of 400 cases with mediastinal cysts and neoplasms, indicating that mesenchymal origin consists of $6 \%$ of all. Less frequency of leiomyoma of the mediastinum is easy to be supposed. Considering these facts, the present paper will be informative to clinicians both in making clinical diagnosis and in selection of therapeutic procedures for leiomyoma of mediastinum origin. 


\section{References}

I) Davis, R.D., Oldham, H.N. \& Sabiston, D.C. (1987) Primary cysts and neoplasms of the mediastinum: Recent changes in clinical presentation, methods of diagnosis, management, and results. Ann. thorac. Surg., 44, 229-237.

2) Morrison, I.M. (1958) Tumors and cysts of the mediastinum. Thorax, 13, 294-307.

3) Nishimoto, M., Yamada, H., Mastumoto, S. \& Oshima, H. (1957) Huge leiomyoma (fibroleiomyoma) originated in the right posterior superior mediastinum. Report of a case. Nippon Geka Hokan, 26, 464-468. (in Japanese with English abstract)

4) Petrikova, J. (1982) Mediastinal tumors and congenital malformations - diagnosis and therapy. Acta Univ. Carol. Med. (Praha), 28, 387-471.

5) Rasaretnam, R. \& Panabokke, R.G. (1975) Leiomyosarcoma of the mediastinum. Brit. J. Dis. Chest., 69, 63-69.

6) Suzuki, T., Ishihara, T., Yamazaki, S., Takanami, I., Fukai, S. \& Hanaoka, K. (1982) Vascular-leiomyoma of the posterior mediastinum. A case report. Jap. J. thorac. Dis., 20, 1075-1078. (Japanese) 